\title{
What not to do in acute otitis media: the top five recommendations proposed by the Italian Society of Preventive and Social Pediatrics
}

\author{
Elena Chiappini, Barbara Bortone, Mattia Doria, Massimo Landi, Giuseppe Di \\ Mauro \& Paola Marchisio
}

To cite this article: Elena Chiappini, Barbara Bortone, Mattia Doria, Massimo Landi,

Giuseppe Di Mauro \& Paola Marchisio (2017): What not to do in acute otitis media: the top five recommendations proposed by the Italian Society of Preventive and Social Pediatrics, Expert Review of Anti-infective Therapy, DOI: 10.1080/14787210.2017.1380518

To link to this article: http://dx.doi.org/10.1080/14787210.2017.1380518

Accepted author version posted online: 18

Sep 2017.

Submit your article to this journal ¿

View related articles ¿ð

View Crossmark data ד 


\section{Special report}

What not to do in acute otitis media: the top five recommendations proposed by the Italian Society of Preventive and Social Pediatrics

Elena Chiappini ${ }^{1}$, Barbara Bortone ${ }^{1}$, Mattia Doria ${ }^{2}$, Massimo Landi ${ }^{3}$, Giuseppe Di Mauro ${ }^{4}$, Paola Marchisio $^{5}$

${ }^{1}$ Anna Meyer Children's University Hospital, Department of Health Sciences, Section of

Paediatrics, University of Florence, Florence, Italy

${ }^{2}$ Primary care pediatrician, Venice, Italy

${ }^{3}$ Primary care pediatrician, Turin, Italy

${ }^{4}$ Primary care pediatrician, Caserta, Italy, President of the Italian Society of Preventive and Social Pediatrics (SIPPS)

${ }^{5}$ Pediatric Highly Intensive Care Unit, Department of Pathophysiology and Transplantation, Università degli Studi di Milano, Fondazione IRCCS Ca' Granda Ospedale Maggiore, Milan, Italy

*Corresponding author:

Elena Chiappini

VialePieraccini, 24, Florence, 50100 Italy

Email: Elena.chiappini@unifi.it

Phone: 00390555662480

Fax: 00390555662900 


\begin{abstract}
Introduction: With the aim to reduce inappropriate procedures and antibiotic therapy in the management of acute otitis media (AOM) in children, the Italian Society of Preventive and Social Pediatrics (SIPPS) proposed a top five list of recommendations for clinical practice.

Areas covered: AOM is one of the most frequent reasons for antibiotic prescription in pediatric age. The over-estimation of AOM is associated with inappropriate treatment, increased costs, adverse events and spread of antibiotic resistance. Thus, the most recent guidelines provided stringent diagnostic criteria and considered the "watchful waiting" approach, limiting the immediate antibiotic therapy to a well-characterized subgroup of children.

Expert commentary: The five recommendations proposed are: 1) Do not diagnose AOM without having documented the presence of middle ear effusion 2) Do not diagnose AOM without examining the entire tympanic membrane; 3) Do not treat immediately all cases of AOM with antibiotics; 4) Do not administer ear analgesic drops until examining the whole tympanic membrane 5) Do not use macrolides in the AOM therapy. This list of top five recommendations could be a novel tool to spread the key messages on the guidelines and to promote the correct diagnostic procedures as well as a rational use of antibiotics in children.
\end{abstract}

Keywords: Acute otitis media, Antibiotics, Antimicrobial resistance, Children, Otoscope 


\section{Introduction}

Antibiotics are among the drugs most commonly prescribed to children. It has been reported that approximately $60 \%$ of children in hospital settings (1-2) and $20 \%$ of children in ambulatory visits receive at least one antibiotic (3). Unfortunately, a great number of these antibiotic prescriptions are unnecessary or inappropriate. Improper antibiotic therapy is a leading factor in wasting health care resources and places patients at risk of developing adverse events and especially antimicrobial resistance. Therefore, the implementation of strategies to preserve the activity of antimicrobial agents has become an urgent public health priority. In this regard the American Academy of Pediatrics (AAP) drew up a list of 10 recommendations to be adopted in pediatric clinical practice (4). The first of these messages discouraged the use of antibiotics in cases of apparent viral respiratory infections, because of the frequent resulting adverse events such as diarrhea and rash and especially of the spread of resistant bacterial strains (4). Moreover, many scientific societies and government agencies joined antimicrobial stewardship initiatives that have been shown to be effective in reducing the inappropriate use of antibiotic therapy (5).

In particular, $\mathrm{AOM}$ is one of the most frequent pediatric diseases: more than $80 \%$ of children under 3 years of age present at least one episode of AOM and about one-third of children has a recurrence (6-7). In Europe, the incidence of AOM in outpatient care is 268 episodes per 1000 people-year (CI 95\%: 254-283) (8) and in Italy, in children under 5 years of age, is around 16\% (9). Thus, AOM is one of the most frequent indications for antibiotic therapy in children. The proportion of children with AOM treated with antibiotics ranges from about 50\% in Germany (10), to $65 \%$ in the Netherlands (11), up to $80 \%$ in the US (12) and in Italy (13).

Therefore, a team of experts in the management of the child's AOM from the Italian Society for Prevention and Social Pediatrics (SIPPS) has developed and proposed a top five list of recommendations on the management of AOM.

\section{Methodology}

A panel of experts identified, through a review of literature and consensus, five items for the list of recommendations. Once obtained the consent on the final list of five items, the statements were formulated. Drafted recommendations were formulated and then reviewed and discussed within the panel, until a final consensus was reached. Each message presented as a single, action-oriented sentence and is followed by an overview of the evidence behind the recommendation. 


\section{Results}

The five most relevant messages have been summarized in Table 1.

\subsection{First message}

\section{Do not diagnose AOM without having documented the presence of middle ear effusion}

International literature indicates a high number of errors in diagnoses of AOM and in particular the high over-estimation of AOM frequency, often diagnosed in cases of merely hyperemic tympanic membrane or in children with otitis media with effusion (OME) $(14,15)$. In order to avoid unnecessary and unjustified treatments it is therefore imperative to differentiate carefully subjects with AOM from those with OME or other forms $(16,17)$.

The diagnosis of AOM cannot be based only on symptoms that are non-specific and overlapping with those of other upper respiratory tract infections. The key diagnostic element of AOM is the presence of middle ear fluid, as shown by cultures of samples obtained by tympanocentesis (18).

The diagnostic criteria of AOM are the moderate to severe bulging of the tympanic membrane, the acute onset acute otorrhea not due to acute external otitis, the slight bulging associated with a recent onset (within 48 hours) of otalgia (ear tugging/rubbing/holding, excessive crying in the youngest child) or intense membrane hyperemia $(16,17)$. The standard tool recommended by guidelines $(16,17)$ in diagnosing AOM is the pneumatic otoscope, which can detect the presence of endotympanic exudate more significantly than the simple otoscope. Pneumatic otoscopy allows the assessment of the contour of the TM, its color, its translucency and its mobility.

To perform a correct pneumatic otoscopy, clinicians should firstly select the speculum size that best fits the external ear canal. It is necessary to assure that the pneumatic system is leak-free by squeezing and releasing the bulb against a fingertip, in order to confirm an effective suction $(16,17)$. With the aim of getting a better view of the tympanic membrane, pediatricians should gently pull the ear backward to straighten the ear canal. The otoscope should be inserted far enough to create a good seal and to prevent air leakage between the speculum and ear canal wall. Attention should be paid not to insert the device too deeply. The ear canal and the eardrum should be carefully explored assessing color, translucency, and position of the tympanic membrane (16,17). A normal tympanic membrane is convex, translucent, and intact. The pneumatic procedure consists of gently squeezing the bulb in order to create positive pressure on the tympanic membrane and to observe the degree of its mobility. Subsequently, the bulb should be released to create negative pressure on the tympanic membrane and observe the degree of TM mobility.

Inward movement of the TM on positive pressure in the external canal, and outward movement on negative pressure should occur, especially in the superior posterior quadrant $(16,17)$. If the 
tympanic membrane does not move with applications of slight positive or negative pressure, a middle ear effusion is highly likely.

Therefore, it is essential to ensure adequate instruction in the proper evaluation of the child's middle ear status and to require adequate post-graduate practice (19).

Appropriate tools for learning ear examination can be represented by the use of video-otoscopes and of educational videos available at specific websites suggested by American guidelines (http://www2.aap.org/sections/infectdis/ video, ePROM: Enhancing Proficiency in Otitis Media) $(19,20,21)$. In uncertain cases, if a pneumatic otoscope is not available, the pediatrician could combine a simple otoscopic assessment with tympanometry, or could address the patient to the otorhinolaryngologist that uses optical microscopy, otoendoscopy or impedance measurement (16).

\subsection{Second message}

\section{Do not diagnose AOM without examining the whole tympanic membrane}

The main problem in AOM diagnoses is the difficulty of properly visualizing the tympanic membrane. The otoscopic assessment can be complicated or impeded by cerumen, epidermal debris or any other cause that completely or partially covers the tympanic membrane. Cerumen obstructing at least $50 \%$ of the ear canal has been described in approximately $44 \%$ of children and $52 \%$ of these children were diagnosed with AOM (22). As highlighted in the latest guidelines, the cerumen removal is essential for the proper diagnosis and management of the AOM $(16,17)$. Pediatricians can remove it by irrigation of the ear canal with warm water (with a syringe of 10-20 mL). Alternatively, only if performed by experienced hands and using a surgical head otoscope, it is possible to remove cerumen by using a curette with a loop (Billeau curette) or speculums equipped with a small hook $(16,22)$. Currently, there are plastic curettes illuminated by a small light source and not completely obstructing the ear canal; these devices, even without a necessary specific experience, make it possible to remove the "soft" cerumen. This practice needs head immobilization in order to prevent ear canal skin lesions and bleeding, that may be abundant during crying, and that may obstruct tympanic membrane visualization, other than alert the pediatrician and parents. A few drops of $10 \%$ hydrogen peroxide, instilled and left into the ear canal for two or three minutes, make the cerumen softer. Alternatively, if necessary, cerumen removal may require the intervention of the otorhinolaryngologist. An explanatory video with all the details on removal practice is available on the website of the New England Journal of Medicine (22,23). 


\subsection{Third message:}

\section{Do not treat immediately all cases of AOM with antibiotics.}

Considering the risk-benefit ratio, guidelines recommend the "wait and see" strategy in children aged from 6 months to 2 years with unilateral AOM and mild symptoms and signs and in children of 2 years and older with unilateral AOM (either mild or severe) or bilateral with mild signs and symptoms.

On the other hand, an immediate antibiotic therapy is always recommended in children younger than 2 years with bilateral (both severe and mild) AOM, in children older than 2 years with severe bilateral AOM, in those with othorrea from spontaneous perforation and those with a history of recurrence $(16,17)$. In addition, antibiotic treatment is reasonably advisable in children under 2 years of age with severe symptoms, when a certain AOM diagnosis is not achievable, even performing all diagnostic procedures $(16,17)$.

Metanalysis and controlled randomized trials $(24,25)$ demonstrated a greater benefit of the immediate antibiotic therapy in terms of pain and fever reduction in cases of bilateral AOM in children under 2 years of age and in children with associated othorrea. Compared with subjects with unilateral AOM, cases with bilateral AOM are more likely to be of bacterial etiology $(25,26)$, and the age under two years is associated with slower spontaneous resolution and higher rate of clinical failure (27). This strategy is also confirmed by the results of a recent randomized, double blind, placebo-controlled study including children aged 3 to 35 months with a certain diagnosis of AOM, aimed at assessing the effect of antibiotic therapy on the resolution time of the middle ear effusion. Children were followed-up for 3 months and the comparison between the antibiotic-treated group and the placebo group did not reveal any significant difference in terms of time of middle ear effusion resolution (28).

It is clear that an assessment of the severity of the episode is critical to adequately choose between watchful waiting strategy and immediate antibiotic therapy. For this purpose, different and not overlapping definitions of severity are available (29,30). Mc Cormick et al (25) provided a 3-score score (OM-3) that evaluates symptoms (otalgia, fever), child's suffering (irritability, disapproval) and activity limitation and a 5 Items score (ETG-5) including fever, otalgia, irritability, discomfort and sleep disturbances. Both are able to detect a change in the severity of AOM. Mc Cormick and collaborators also developed a visual acuity scale named Acute Otitis Media-Faces Scale (AOMFS), with faces similar to those of the Wong-Baker Pain Scale, that, if associated with an otoscopic score (OS-8), is related to clinical changes (31). Shaikh et al. validated a seven items (AOM-SOS) score recorded by parents/tutors including ear touching or rubbing, crying, irritability, difficulty in sleeping, reduced activity or appetite, and fever (32). This score was related to otoscopic diagnoses 
(AOM, OME, and normal middle ear status) and appropriately changed according to the clinical evolution (32). These scales have the limit of not attributing to each item a different weight based on its prognostic value. At this regard, it is interesting to point out the scale proposed by the Japanese guideline (33), which assigns different scores to the following parameters: age, otalgia intensity, fever, crying, hyperemia or bulging of the tympanic membrane, otorrhea. Even if the Japanese score seems to be a more versatile tool, at the moment there is no evidence of superiority of a severity score on the others.

The "wait and see" strategy should be adopted only if an adequate follow-up is feasible. Moreover, antibiotic should be prescribed in children with clinical worsening or not improving within 48 to 72 hours $(16,17)$. Parents/tutors should be advised about the benign and self-limiting nature of most AOMs. (16,17). The follow-up procedure has not been yet standardized and remains at the discretion of the physician, who may decide to schedule a check-up visit, establish a telephone contact, or prescribe an antibiotic therapy to be administered in case of clinical worsening. A randomized placebo-controlled Finnish study on children aged 3 to 35 months with AOM diagnosis showed that changes in otoscopic MT appearance were related $(86 \%)$ to the parental clinical report (AOM-SOS). Therefore, children with reported clinical improvement may not need a routine checkup visit but a phone follow-up may be sufficient (34).

\subsection{Fourth message}

\section{Do not administer ear analgesic drops until examining the whole tympanic membrane}

The management of AOM should include assessment and treatment of otalgia. Paracetamol or ibuprofen could be used $(16,17,35,36)$. The use of anesthetic eardrops can be considered only in combination with systemic analgesic therapy, in case of moderate to severe otalgia, as they have the advantage of controlling pain more rapidly than systemic analgesics. Analgesic drops can be applied only after tympanic membrane assessment and after excluding a tympanic perforation as they may complicate global tympanic membrane visualization (16).

\subsection{Fifth message}

\section{Do not use macrolides in the AOM therapy}

The 3 most common bacterial pathogens in AOM are Streptococcus pneumoniae, Haemophilus influenzae and Moraxella catarrhalis. Therefore, amoxicillin or amoxicillin plus clavulanic acid are 
recommended as first-line treatment.In case of uncomplicated AOM and mild symptoms in absence of risk factors for bacterial resistance and without a history of recurrence, the first choice therapy is amoxicillin (80-90 mg/ kg/day). In children with complicated AOM with severe symptoms and / or in subjects at risk for bacterial resistance, or with a history of recurrence, who have taken amoxicillin in the previous 30 days, therapy should be initiated with high-dose amoxicillinclavulanate $(80-90 \mathrm{mg} / \mathrm{kg} /$ day of amoxicillin) (16).

A potential strategy for reducing the spread of antibiotic resistance could be to shorten the duration of therapy below the standard 10 days $(17,37)$. Studies comparing the efficacy of 10 days antibiotic therapy (amoxicillin, trimethoprym sulphamethoxazole, cephalosporin and macrolides) with that of 5 -7 days regimen did not show any significant differences except for a minimal superiority of prolonged duration in children younger than 2 years (37) or in children with history of AOM in the previous month or at risk of bacterial resistance (38). A 2010 Cochrane review on evaluating standard duration therapy with azithromycin and ceftriaxone versus a 5-7 day-regimen, demonstrated a minimal short-term superiority of standard duration but a higher risk of side effects (39).

Hoberman et al. (27) compared, in children 6 to 23 months of age with certain diagnosis of AOM, the efficacy of antibiotic therapy with amoxicillin-clavulanic acid at $90 \mathrm{mg} / \mathrm{kg}$ for 10 days and efficacy of 5 day-therapy considering the percentage of clinical failure, incidence of resistant strains, adverse effects, recurrence rate and persistence of middle ear fluid. There was a greater percentage of clinical failure in children treated for 5 days ( 77 of 229 children [34\%] vs. 39 of 238 [16\%]) with a difference of $17 \%$ (27). On the other hand, there was no significant difference between the two groups in terms of risk of monthly recurrence, of residual middle ear effusion frequency, of adverse events, and especially in terms of the onset of resistant bacteria during or after therapy (27).

Therefore, antibiotic therapy should last 10 days in children at risk of worsening (less than 2 years and / or with spontaneous otorrhea), while 5 days therapy may be adopted in children younger than 2 years without risk factors for unfavorable evolution $(16,27)$.

Macrolides have limited efficacy against AOM pathogen bacteria (S.pneumoniae, H.influenzae, M. catarrhalis), since antibiotic resistance rates widely varies between countries and in Europe a figure of $26 \%$ for S. pneumoniae and $38 \%$ for S. pyogenes is described (40). Macrolides should be used only in children allergic to penicillins. Cross reactivity between penicillin and second or third generation cephalosporins is negligible, due to the different chemical structure (41). On the other hand, it has been shown that the use of third generation cephalosporins leads to the selection of resistant strains such as extended-spectrum beta lactamase (ESBL) Enterobacteriaceae $(42,43)$. 
Therefore, if allergic tests are not available, when there is no history of severe or recent allergic reaction, second generation cephalosporins could be recommended. Macrolides should, therefore, be administered only in cases of severe allergy to penicillins and cephalosporins (16).

\section{Conclusions}

The main objective of proposing these five messages is to promote a correct use of antibiotics in AOM management. The therapeutic choice is possible only after visualization of the entire tympanic membrane with the technique and instrumentation recommended by the guidelines. Antibiotics should be used only when watchful waiting strategy is not feasible such as in children younger than 2 years with bilateral otitis, in children older than 2 years with serious bilateral symptoms, in those with otorrhea and with recurrence history.

\section{Expert commentary}

The key weaknesses in the management of AOM are the difficulties of distinguishing AOM from OME from TM hyperemia, and of selecting patients who need antibiotic therapy from those eligible for the watchful waiting strategy. Moreover, there are not standardized AOM severity scores or a definite follow-up procedure by pediatricians. Recent guidelines have established rigorous diagnostic criteria and have offered precise categories of patients to guide the choice between immediate antibiotic therapy and watchful waiting strategy $(16,17)$. Such selection results from consideration of patient's characteristics such as age, bilateral or monolateral AOM, clinical severity, epidemiology, prior antibiotic therapy. Unfortunately, little adherence to the guidelines' recommendations has been reported, as shown by the high percentage of antibiotic prescription (44). Therefore, it is essential to provide physicians with readily available tools to facilitate the acquisition of diagnostic and therapeutic criteria. This list of recommendations could be a valuable instrument thanks to its synthetic ability and clear presentation of supporting evidences. It is also essential to institute education courses on the inspection of the tympanic membrane, the use of the pneumatic otoscope, the impedance-measuring device other than the technique to remove cerumen

from the external auditory duct. Nevertheless, incisive campaigns to promote parents' awareness of proper antibiotic should be warranted.

When an antibiotic therapy is required, the first choice drug should be amoxicillin $(80-90 \mathrm{mg} /$ $\mathrm{kg}$ /day) or high-dose amoxicillin-clavulanate $(80-90 \mathrm{mg} / \mathrm{kg} /$ day of amoxicillin), in cases at risk of recurrence or resistance. Education programs and antibiotic stewardship campaigns should focus on reducing the inappropriate use of macrolides and of third generation cephalosporins because of 
selection of resistant strains. Scientific research in this field should be aimed at providing further evidence on the safety of the observation approach, at standardizing a valid and simple severity score and a definite follow-up strategy.

The most recent research has focused on establishing the appropriate duration of therapy (37-39). Several studies compared standard duration therapy with lower duration therapy. The results were discordant and did not show significant differences unless a minimum superiority of prolonged duration in children under the age of 2 years (37) or in children with history of AOM in the previous month or at risk of resistant infections (38). Interestingly, Hoberman's 2016 study (27) showed higher percentage of clinical failure in children treated for 5 days without significant differences about the side effects and the occurrence of resistant bacteria either in course or after the therapeutic cycle. Therefore, the certain effective strategies able to contain antibiotic resistance are the correct diagnosis of AOM and the precise selection of the patients to treat but not to shorten therapy duration, unless in very limited cases, whose definition still needs further studies.

Current research is also focusing on validating severity scores of AOM and follow up strategies. A randomized placebo-controlled Finnish study (34) on children aged 3 to 35 months with AOM showed that the improvement in the otoscopic examination findings correlates well (86\%) with the clinical report described by parents with the AOM-SOS score.

Therefore, in children with clinical improvement reported by parents, a routine scheduled follow-up appointment may not be required, but a telephone follow-up may be sufficient (34).

A recent Cochrane review has evaluated the effects of advising a delayed prescription of antibiotics in respiratory tract infections (including three studies on AOM) on clinical outcomes, antibiotic use, antibiotic resistance and patient satisfaction (45). The study has shown that a delayed antibiotics strategy may be an acceptable compromise in place of immediate prescribing to significantly reduce unnecessary antibiotic use for RTIs, and thereby reduce antibiotic resistance, while maintaining patient safety and satisfaction levels (45).

\section{Five-year view}

Looking forward over the next 5 years, significant advances are expected in clinical research in these regards that may extensively impact the quality of AOM management.

A further diffusion in the everyday clinical practice of pneumatic otoscopy and of cerumen removal is desirable. Indeed, we believe that the standardization of severity scores and follow up procedures could give greater solidity and security to the watchful waiting strategy leading to greater adherence to this approach. 


\section{Key issues}

- $\mathrm{AOM}$ is one of the most frequent indications for antibiotic therapy in children. Unfortunately, a great number of these antibiotic prescriptions are unnecessary or inappropriate.

- In order to avoid unjustified treatments it is imperative to differentiate carefully subjects with AOM from those with OME or other forms.

- The key diagnostic element of AOM is the presence of middle ear fluid. The standard tool recommended by guidelines in diagnosing AOM is the pneumatic otoscope.

- With the aim of visualizing the whole tympanic membrane it is essential to remove cerumen from the external auditory canal.

- The "wait and see" strategy is recommended in children aged from 6 months to 2 years with unilateral AOM and mild symptoms and signs and in children of 2 years and older with unilateral AOM (either mild or severe) or bilateral with mild signs and symptoms.

- The "wait and see" strategy should be adopted only if an adequate follow-up is feasible.

- Analgesic drops can be applied only after tympanic membrane assessment and after excluding a tympanic perforation.

- Macrolides have limited efficacy against AOM pathogen bacteria. Therefore, they should be administered only in cases of severe allergy to penicillins and cephalosporins.

\section{Funding}

The manuscript was not funded.

\section{Declaration of interest}

The authors have no relevant affiliations or financial involvement with any organization or entity with a financial interest in or financial conflict with the subject matter or materials discussed in the manuscript. This includes employment, consultancies, honoraria, stock ownership or options, expert testimony, grants or patents received or pending, or royalties. 


\section{References}

Reference annotations

* Of interest

** Of considerable interest

1. Levy ER, Swami S, Dubois SG, Wendt R, Banerjee R. Rates and appropriateness of antimicrobial prescribing at an academic children's hospital, 2007-2010. Infect Control Hosp Epidemiol. 2012.33:346-53

2. Grohskopf LA, Huskins WC, Sinkowitz-Cochran RL, Levine GL, Goldmann DA, Jarvis WR. Use of antimicrobial agents in United States neonatal and pediatric intensive care patients. Pediatr Infect Dis J. 2005.24:766-73.

3. Hersh AL, Shapiro DJ, Pavia AT, Shah SS. Antibiotic prescribing in ambulatory pediatrics in the United States. Pediatrics. 2011.128:1053-61.

4. American Academy of Pediatrics. Ten Things Physicians and Patients Should Question. http://www.choosingwisely.org/ societies/american-academy-of-pediatrics .

5. Gerber JS, Prasad PA, Fiks AG et al. Effect of an outpatient antimicrobial stewardship intervention on broad-spectrum antibiotic prescribing by primary care pediatricians: a randomized trial. JAMA. 2013. 12. 309:2345-52*

\section{* A study that describes the impact of antimicrobial stewardship campaigns on pediatricians therapeutic choices.}

6. Rovers MM, Schilder AGM, Zielhius GA, Rosenfeld RM. Otitis media. Lancet. 2004. 363:465473.

7. Pelton SI. Otitis media: Re-evaluation of diagnosis and treatment in the era of antimicrobial resistance, pneumococcal conjugate vaccine, and evolving morbidity. PediatrClin North Am 2005. 52:711-728.

8. Liese J, Carmona A, Cantarutti L, et al. Incidence of acute otitis media in young childrenseen in European medical practices. 6th World Congress of the World Society for Pediatric Infectious Diseases. 2009

9. Marchisio P, Cantarutti L, Sturkenboom M, et al. Burden of acute otitis media in primary care pediatrics in Italy: a secondary data analysis from the Pedianet database.Pedianet. BMC Pediatr. 2012. 12:185. 
10. Schnabel E, Sausenthaler S, Brockow I, et al. Burden of otitis media and pneumonia in children up to 6 years of age: results of the LISA birth cohort. Eur J Pediatr. 2009.168:1251-7.

11. Plasschaert AI, Rovers MM, Schilder AG, Verheij TJ, Hak E. Trends in doctor consultations, antibiotic prescription, and specialist referrals for otitis media in children: 1995-2003. Pediatrics 2006. 117:1879-86.

12. Grijalva CG, Nuorti JP, Griffin MR. Antibiotic prescription rates for acute respiratory tract infections in US ambulatory settings. JAMA 2009;19;302:758-66.

13. Moro ML, Marchi M, Gagliotti C, Di Mario S, Resi D; "Progetto Bambini a Antibiotici [ProBA]" Regional Group. Why do paediatricians prescribe antibiotics? Results of an Italian regional project. BMC Pediatr. 2009. 9:69.

14. Pichichero ME, Casey JR. Diagnostic inaccuracy and subject exclusions render placebo and observational studies of acuteotitis media inconclusive. Pediatr Infect Dis J. 2008. 27:958-62.

15. Paradise JL. On classifying otitis media as suppurative or nonsuppurative, with a suggested clinical schema. J Pediatr. 1987.111:948-51

16. Marchisio P, Bellussi L, Di Mauro G, et al. Acute otitis media: From diagnosis to prevention. Summary of the Italian guideline.Int J Pediatr Otorhinolaryngol. 2010. 74:1209-16**

**Clinical practice guidelines about AOM diagnoses and therapy in children (16-17).

17. Lieberthal AS, Carroll AE, Chonmaitree T et al. The diagnosis and management of acute otitis media.Pediatrics. 2013.131:964-99*

18. McCormick DP, Lim-Melia E, Saeed K, Baldwin CD, Chonmaitree T. Otitis media: can clinical findings predict bacterial or viral etiology? PediatrInfect Dis J. 2000. 19:256

19. Marchisio P, Mira E, Klersy C, et al. Medical education and attitudes about acute otitis media guidelines: a survey of Italian pediatricians and otolaryngologists. PediatrInfect Dis J. 2009.28: $1-4$

20. Kaleida PH, Ploof D. ePROM: Enhancing Proficiency in Otitis Media. Pittsburgh, PA: University of Pittsburgh School of Medicine. Available at: http://pedsed.pitt.edu.

21. Innovative Medical Education. A View Through the Otoscope: Distinguishing Acute Otitis Media from Otitis Media with Effusion. Paramus, NJ: Innovative MedicalEducation; 2000

22. Marchisio P, Pipolo C, Landi M et al. Cerumen: A fundamentalbutneglectedproblem by pediatricians; ItalianEarwaxStudyGroup.Int J PediatrOtorhinolaryngol. 2016. 87:55-60*

* Study that explains the importance of cerumen removal and provides precise practical instructions of the procedure. 
23. Shaikh N., Hoberman A., Kaleida P.H:, Ploof D.L., Paradise J.L . Videos in clinical medicine. Diagnosing otitis media-otoscopy and cerumen removal. N Engl J Med. 2010. 362: 62

24. Rovers MM, Glasziou P, Appelman $\mathrm{Cl}$ et al. Antibiotics for acute otitis media: a meta analysis with individual patient data. Lancet 2006. 368:1429-1435

25. McCormick DP, Chonmaitree T, Pittman C, et al. Nonsevere acute otitis media: a clinical trial comparing outcomes of watchful waiting versus immediate antibiotic treatment. Pediatrics. 2005.115: $1455-1465^{* *}$

** An important study comparing outcomes of "wait and see" procedure versus immediate antibiotic treatment.

26. Palmu A, Herva E, Savolainen H, Karma P, Makela H, Kilpi TM. Association of clinical signs and symptoms with bacterial findings in acute otitis media.CID.2004. 38:234-242

27. Hoberman A, Paradise JL, Rockette HE, et al. Shortened Antimicrobial Treatment for Acute Otitis Media in Young Children. N Engl J Med. 2016. 22.375: 2446-2456. **



28. Ruohola A, Laine MK, Tähtinen PA. Effect of Antimicrobial Treatment on the Resolution of Middle-Ear Effusion After Acute Otitis Media. J Pediatric Infect Dis Soc. 2017J Pediatric Infect Dis Soc. 2017 Mar 16. doi: 10.1093/jpids/pix008. [Epub ahead of print]

29. Hotomi M, Yamanaka N, Samukawa T, et al. Treatment and outcome of severe and non-severe acute otitis media. Eur J Pediatr 2005. 164:3-8

30. Le Saux N, Gaboury I, Baird M, et al. A randomized, double-blind, placebo-controlled noninferiority trail of amoxicillin for clinically diagnose acute otitis media in children 6 months to 5 years of age. CMAJ 2005. 172:335-341.

31. Friedman NR, McCormick DP, Pittman C, et al. Development of a practical tool for assessing the severity of acute otitis media. Pediatr Infect Dis J. 2006. 25.101- 107

32. Shaikh N, Rockette HE, Hoberman A, Kurs-Lasky M, Paradise JL. Determination of the minimal important difference for the acute otitis media severity of symptom scale. PediatrInfect Dis J. 2015.34:41-3.

33. Kitamura K, Iino Y, Kamide Y, Kudo F, Nakayama T, Suzuki K, Taiji H, Takahashi H, Yamanaka N, Uno Y. Clinical practice guidelines for the diagnosis and management of acute otitis media (AOM) in children in Japan - 2013 update. Auris Nasus Larynx. 2015 . 42:99-106. 
34. Uitti JM, Tähtinen PA, Laine MK, Ruohola A, Close Follow up in children with acute otitis media initially managed without antimicrobials. JAMA Pediatr. 2016 .1.170:1107-1108.

35. Sjoukes A, Venekamp RP, van de Pol AC, Hay AD, Little P, Schilder AG, Damoiseaux RA. Paracetamol (acetaminophen) or non-steroidal anti-inflammatory drugs, alone or combined, for pain relief in acute otitis media in children.Cochrane Database Syst Rev. 2016 15.12.

36. Ovnat Tamir S, Shemesh S, Oron Y, Marom T. Acute otitis media guidelines in selected developed and developing countries uniformity and diversity. Arch Dis Child. 2016

37. Cohen R, Levy C, Boucherat M, et al. Five vs. ten days of antibiotic therapy for acute otitis media in young children. Pediatr Infect Dis J 2000.19:458-463*

*A series of three publications (37-38-39) about efficacy of short vs stardand duration antibiotic therapy of acute otitis media.

38. Pichichero ME, Marsocci SM, Murphy ML, Hoeger W, Francis AB, Green JL. A prospective observational study of 5-, 7-, and 10-day antibiotic treatment for acute otitis media. Otolaryngol Head Neck Surg. 2001.124:381-387*

39. Kozyrskyj A, Klassen TP, Moffatt M, Harvey K. Short-course antibiotics for acute otitis media. Cochrane Database Syst Rev 2010.CD001095*

40. Stefani S, Mezzatesta ML, Fadda G et al. Antibacterial activity of cefditoren against major community-acquired respiratory pathogens recently isolated in Italy. J Chemother. 2008.20:5619.

41. Pichichero ME. Use of selected cephalosporins in penicillin-allergic patients: a paradigm shift. DiagnMicrobiol Infect Dis. 2007. 57:13-18

42. Logan LK, Braykov NP, Weinstein RA, Laxminarayan R; CDC Epicenters Prevention Program. Extended-Spectrum $\beta$-Lactamase-Producing and Third-Generation Cephalosporin-Resistant Enterobacteriaceae in Children: Trends in the United States, 1999-2011. J Pediatric Infect Dis Soc. 2014. 3:320-8.

43. Zerr DM, Miles-Jay A, Kronman MP et al. Previous Antibiotic Exposure Increases Risk of Infection with Extended-Spectrum- $\beta$-Lactamase- and AmpC-Producing Escherichia coli and Klebsiella pneumoniae in Pediatric Patients. Antimicrob Agents Chemother. 2016. 20.60:423743.

44. Palma S, Rosafio C, Del Giovane C et al. The impact of the Italian guidelines on antibiotic prescription practices for acute otitis media in a paediatric emergency setting. Ital $\mathrm{J}$ Pediatr. 2015. $41: 37$ 
45. Spurling GK, Del Mar CB, Dooley L, Foxlee R, Farley R. Delayed antibiotic prescriptions for respiratory infections. Cochrane Database Syst Rev. 2017. 9: CD004417. 


\begin{tabular}{|c|c|}
\hline \multirow[b]{2}{*}{ 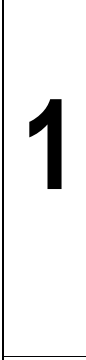 } & $\begin{array}{l}\text { Do not diagnose AOM without having documented the presence ofmiddle ear } \\
\text { effusion }\end{array}$ \\
\hline & $\begin{array}{l}\text { Diagnosis of AOM cannot be based only on symptoms, that are non-specific and overlapping with } \\
\text { those of other upper respiratory tract infections. In addition, fever can also be present in case of otitis } \\
\text { media with effusion (OME). The presence of middle ear effusion should always be searched with a } \\
\text { pneumatic otoscope. Accurate signs of middle ear fluid are the clear tympanic membrane bulging or } \\
\text { the spontaneous perforation otorrhea. }\end{array}$ \\
\hline \multirow[b]{2}{*}{2} & Do not diagnose $A O M$ without examining the whole tympanic membrane \\
\hline & $\begin{array}{l}\text { With the aim of visualizing the whole tympanic membrane and its main features (integrity, position, } \\
\text { color, transparency, brightness, and mobility)it is essential to remove cerumen from the external } \\
\text { auditory canal. Cerumen removal can be carried out by adequately trained pediatricians or by an } \\
\text { otorhinolaryngologist with different procedures depending on the operator's training and from the } \\
\text { available tools. }\end{array}$ \\
\hline \multirow[b]{2}{*}{3} & Do not treat immediately all cases of AOM with antibiotics. \\
\hline & $\begin{array}{l}\text { It is recommended to adopt an initial clinical "wait and see" strategy for children aged from } 6 \text { months } \\
\text { to } 2 \text { years with unilateral AOM and mild symptoms and signs and in children of } 2 \text { years and older with } \\
\text { unilateral AOM (either mild or severe) or bilateral with mild signs and symptoms. The watchful waiting } \\
\text { approach is feasible only if previously shared with parents and if it is possible to ensure an adequate } \\
\text { follow-up, at least telephonic, within } 48 \text { to } 72 \text { hours after the onset of symptoms. }\end{array}$ \\
\hline & $\begin{array}{l}\text { Do not administer ear analgesic drops until examining the whole tympanic } \\
\text { membrane }\end{array}$ \\
\hline & $\begin{array}{l}\text { It is not advisable to administer ear analgesic drops in children under } 3 \text { years of age without a } \\
\text { previous tympanic membrane visualization because there is not a solid evidence of efficacy, it is not } \\
\text { safe in case of spontaneous perforation, and may alter the otoscopic assessment because of cerumen } \\
\text { fluidification. }\end{array}$ \\
\hline \multirow{2}{*}{5} & Do not use macrolides in the AOM therapy \\
\hline & $\begin{array}{l}\text { The most common bacterial pathogens in AOM are Streptococcus pneumoniae, Haemophilus } \\
\text { influenzae and Moraxella catarrhalis. These germs show moderate-high sensitivity to betalactams } \\
\text { (mainly amoxicillin, amoxicillin-clavulanic acid and second-generation cephalosporins) but a medium } \\
\text { to high resistance to macrolides. }\end{array}$ \\
\hline
\end{tabular}

Table 1. Top five list in AOM management in children 